\title{
Mathematik muss man fühlen
}

\author{
Michael Röckner im Gespräch mit den Mitteilungen
}

\begin{abstract}
Im Interview spricht Michael Röckner, der scheidende Präsident der DMV, über seine Forschung, die DMV, China, die Mathematik in Deutschland und wie er das alles unter einen Hut gebracht hat.
\end{abstract}

Lieber Herr Röckner, Sie sind Sprecher des Bielefelder Sonderforschungsbereichs 1283 . Sie waren bis vor Kurzem Dekan. Sie pflegen als Wissenschaftler einen intensiven internationalen Austausch. Gelangweilt waren Sie nicht, oder?

Nein, sicher nicht.

Dennoch sind Sie zu allem Überfluss Präsident der DMV geworden. Warum?

Ich habe eine lange Verbundenheit mit der DMV. Ich war für acht Jahre im Präsidium. Dadurch habe ich miterlebt, wie viel Arbeit mit dem Präsidentenamt verbunden ist. Auch frühere Präsidenten, die ich persönlich gut kenne, haben mir das immer deutlich gesagt. Also dachte ich mir, wenn sie dich wirklich einmal fragen sollten, musst du dir das sehr gut überlegen.

Und als man Sie dann gefragt hat?

Da habe ich sofort zugesagt. Präsident der Deutschen Mathematiker-Vereinigung zu sein ist eine Sache - die kann man nicht ablehnen. Wenn man bedenkt, welche beeindruckenden Mathematiker Präsident waren: Cantor, Hilbert, Klein. Mit diesen Mathematikern gewissermaßen in einer Reihe stehen zu dürfen, dazu kann man nicht Nein sagen.

Ist es denn wirklich so viel Arbeit?

Es ist schon ein Knochenjob. Es gibt viele, die einen großen Beitrag für die DMV leisten. Aber am Ende hält der Präsident das Ganze in der Hand und die Hauptlast liegt bei ihm.

Was hat Ihnen am meisten Freude bereitet?

Die Gelegenheit mit Menschen in Kontakt zu kommen, die an Mathematik interessiert sind - egal, ob bei einer Gauss-Vorlesung, bei JugendForscht, bei der MathematikOlympiade oder bei Schulbesuchen. Das war immer stimulierend und für mich eine der schönsten Aufgaben des Amtes. Wenn ich eine Rede halte, ist für mich das Feedback sehr wichtig. Wenn man die Augen auf einen gerichtet sieht und merkt, dass man es schafft einen persönlichen Draht aufzubauen, auch wenn es dreihundert Zuhörer sind.
Bei Mathematik kann man nicht davon ausgehen, dass alle den gleichen Hintergrund haben. Wie schaffen Sie es, das Publikum zu gewinnen, wenn Sie eine Rede für die DMV halten?

Zunächst einmal hatte ich jedes Mal einen echten Bammel, ob ich den Nerv der Zuhörer treffe. Ich versuche, einen persönlichen Bezug zum Anlass, zum Ort und zu den anderen Teilnehmern herzustellen. Zum Beispiel beim Vortrag von Cédric Villani in Regensburg: Cédric kenne ich persönlich schon sehr lange. Zusätzlich habe ich mir angeschaut, was in Regensburg an der Universität und in der Mathematik gerade los ist und ein wenig zur Geschichte der Stadt, insbesondere in Bezug auf die Wissenschaft. Wenn man das alles zusammenbringt, weckt man das Gefühl, dass da nicht abstrakte Personen zufällig ihren Job machen - der eine hält den Vortrag, der andere die Einführung - sondern dass die Mathematik aus Verbindungen zwischen konkreten Mathematikern besteht. Und zu diesen Verbindungen gehört auch der Ort und letztlich das Publikum selbst.

Wie haben Sie den zusätzlichen Aufwand des Präsidentenamtes bewältigt?

Man muss Prioritäten setzen. Die Regel lautete: die DMV geht temporär immer vor. Wenn ich zum Beispiel die Präsidiumssitzung vorbereiten musste - das kostet rund einen Tag - war ich für nichts anderes erreichbar. Das wussten alle und haben auch alle akzeptiert. Danach ging es dann so schnell wie möglich ins Dekanat und dann zurück in den SFB und die Forschung.

Geht das nicht doch zu Lasten der Forschung?

Ja, vieles hat länger gedauert. Beispielsweise habe ich mit vielen Gästen gearbeitet. Manchmal hatte ich ein Gefühl, ein bestimmter Teil in der gemeinsamen Arbeit kann so nicht stimmen. Mathematik muss man ja fühlen. Das ist oft wertvoller, als die Epsilons in einem Beweis zu checken. Dann konnte die Arbeit nicht raus, bis ich die Zeit gefunden hatte, das zu klären. Und das konnte auch mal ziemlich lange dauern.

Können Sie in einer einzelnen freien Stunde Mathematik machen? 


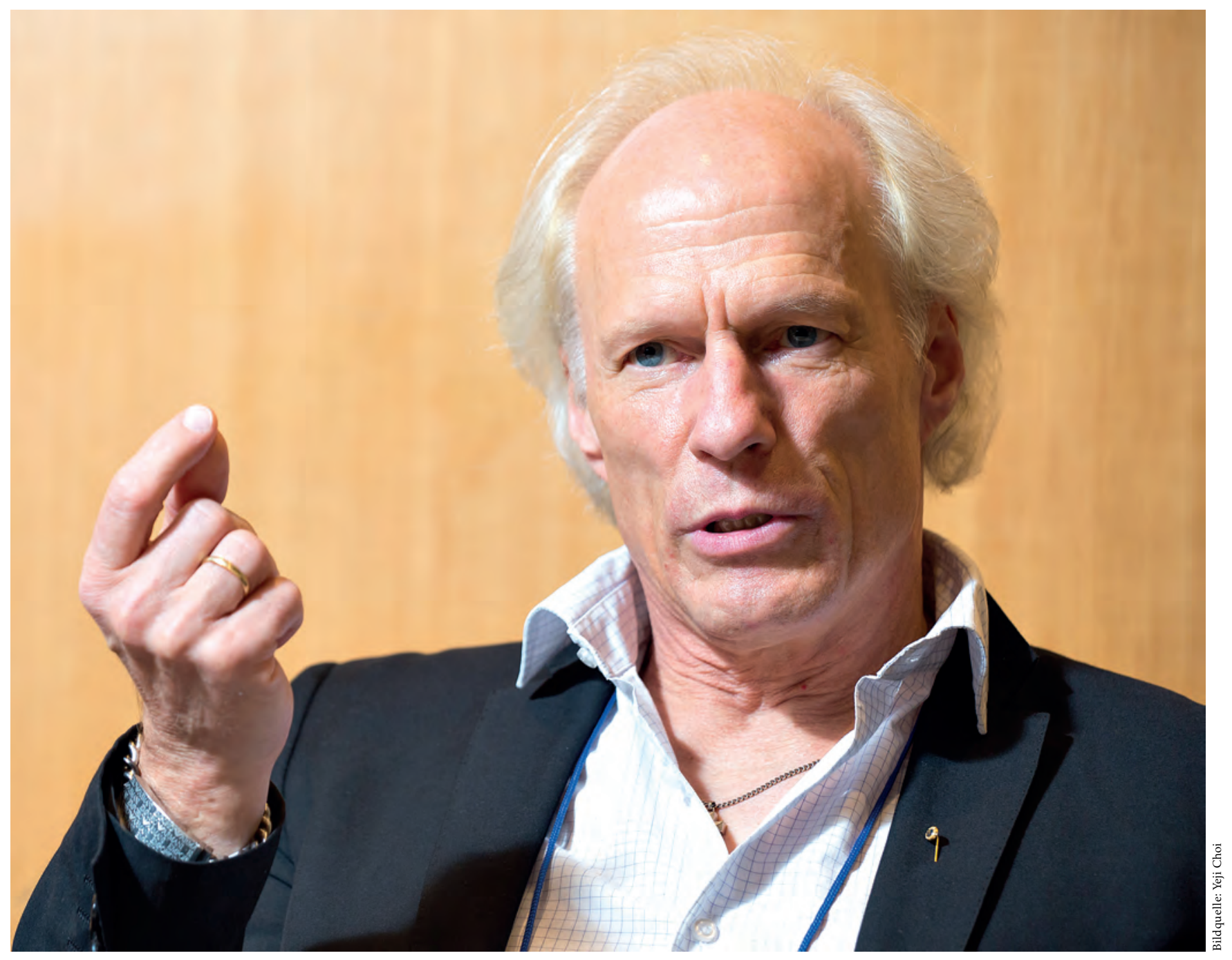

Michael Röckner war von 2017-2018 Präsident der DMV

Nein, wenn ich nur eine Stunde an etwas arbeite, dann muss ich am nächsten Tag wieder von vorne anfangen. Ich brauche mindestens einen Tag, um das richtige Gefühl zu entwickeln und auf eine interessante Spur zu kommen. Und am besten sollte ich dann am nächsten Tag daran weiterarbeiten können.

\section{Gab es auch Dinge, die ganz unter den Tisch fielen?}

Ich habe in der Zeit keine Gutachten angenommen. Auf meinem Laptop gibt es ein kleines Kästchen „apologies“ mit der entsprechenden E-Mail-Vorlage. Für GraduiertenKollegs habe ich eine Ausnahme gemacht, weil es da wirklich schwierig ist, die richtigen Gutachter zu finden. Eine weitere Ausnahme gab es für eine Art Exzellenzcluster in Mathematik bei der Chinesischen Akademie der Wissenschaften. Ich habe dorthin eine fast dreißigjährige Beziehung und hatte es schon vorher zugesagt.

Woher kommt diese lange und intensive Beziehung zu China?
Das begann 1989 mit Zhi-Ming Ma, einem Humboldt Stipendiaten bei Wolfhard Hansen in Bielefeld. Ich war damals noch in Edinburgh. In dieser Zeit lernten Ma und ich uns kennen. Kurz danach haben wir zusammen ein Buch geschrieben [Introduction to the Theory of (Non-Symmetric) Dirichlet Forms, Anm. d. Red.]. Später haben wir mit Sergio Albeverio den Max-Planck-Forschungspreis gewonnen. Dann hat Ma einen hohen Preis in China erhalten und wurde jüngstes Mitglied der Akademie der Wissenschaften - mit 50 Jahren.

Zhi-Ming Ma war auch federführend für die Organisation des ICM 2002 in Peking, richtig?

Ja. Er ist noch heute einer der einflussreichsten Wahrscheinlichkeitstheoretiker in China. Wir hatten ein gemeinsames Internationales Graduiertenkolleg. Ich habe, glaube ich, acht Doktoranden aus China promoviert und hatte eine Reihe von chinesischen Humboldt-Stipendiaten. Viele davon sind recht schnell in China Professor geworden. 
Kann man sagen, dass es sich für die chinesischen Doktoranden ausgezahlt hat, in Bielefeld gewesen zu sein?

Ja, ich glaube, Bielefeld hat einen guten Namen.

Haben Sie über die Mathematik hinaus eine Beziehung zu China und zur chinesischen Kultur entwickelt?

Die persönliche Beziehung kam immer aus und über die Mathematik. Zum Beispiel sind Ma und ich und auch unsere Frauen Freunde geworden. Die chinesische Kultur selbst ist sehr komplex. Man muss lernen, sich darin richtig zu verhalten. Es gibt in China völlig andere Denkmuster. Das hat mich fasziniert.

\section{Was sind das für Denkmuster?}

Es gibt zum Beispiel das Bild „Biege die beiden Enden eines Intervals zu einem Kreis“, wenn man eine Konfliktlösung sucht. Besonders beeindruckt hat mich die Art, im Kopf Konten von Gefälligkeiten zu führen - im besten Sinne - und sich zu bemühen, diese immer im Plus zu haben. Bei uns klingt das negativ: Konten und Gefälligkeiten. Aber in China bedeutet es, dass wenn jemand etwas für mich getan hat - auch professionell - dann versuche ich, den Gefallen mindestens zu erwidern. Sonst fühle ich mich unwohl.

\section{Wie sind Sie mit dieser Haltung umgegangen?}

Mich persönlich betrifft es vor allem bei meinen Promovenden. Die sind mir auf ewig dankbar und wollen es mir gegenüber gut machen. Ich habe ihnen gesagt, das sei einfach mein Job. Es bedürfe keines besonderen Dankes. Aber das stößt auf taube Ohren.

Haben Sie auch leidvolle Erfahrungen mit der kulturellen Differenz gemacht?

Oh ja! Ich habe zusammen mit Ma eine Satellitenkonferenz zum ICM in Peking organisiert. Ich hatte Ma angeschrieben, aber er war zu beschäftigt, um zu antworten. Also habe ich Ma in Ruhe gelassen und ihm ganz am Ende das fertige Programm geschickt.

Das kam nicht gut an?

Ganz und gar nicht! Ma selbst geriet in seiner Gruppe unter Druck, weil einige, die als Sprecher vorgesehen waren, nicht im Programm erschienen, da sie auf keine der Einladungsemails reagiert hatten. Als ich dann nach Peking kam, habe ich mich zunächst sachlich mit den fehlenden Rückmeldungen verteidigt. Das war falsch. Was ich nicht verstanden hatte: so Dinge wie „das Gesicht zu waren“ werden in China noch sensibler gehandhabt als bei uns. Als mir das klar wurde, habe ich schnell eingeräumt, dass wir das Programm früher hätten schicken sollen, und dann konnten wir weiterarbeiten.

Gibt es auch eine andere Kultur des mathematischen Arbeitens?
Zunächst einmal ist der Druck, erfolgreich zu sein, enorm groß. Das gilt besonders für die jungen Leute. An der Akademie sind sie handverlesen und preisbeladen. Sie waren die besten ihrer Provinzen. Und jetzt kommen sie an die Akademie und müssen sich gegen starke Konkurrenz durchsetzen.

\section{Ist das für die Arrivierten anders?}

Ein bisschen. Aber der Druck ist immer noch hoch, zumindest in der Academy of Mathematics and System Science. Das ist sozusagen der Mathematik-Teil der chinesischen Akademie der Wissenschaften. Sie haben sich vorgenommen, in zehn Jahren auf dem Niveau von Princeton konkurrieren zu können, also ein sehr ambitioniertes Ziel im Visier. Das kreiert Druck, sehr effektiv und schnell die nächste Arbeit zu schreiben.

In Ihrer Formulierung liegt etwas Skepsis.

Ma und ich sind da einer Meinung, dass das besonders für junge Mathematiker(innen) der falsche Ansatz ist. Neueste Arbeiten in den Topzeitschriften daraufhin zu lesen, um sie ein wenig $\mathrm{zu}$ verbessern oder recht geradlinig $\mathrm{zu}$ verallgemeinern, sollte nicht Schwerpunkt der Forschung am Anfang der Karriere sein. Es geht darum, tiefliegende mathematische Strukturen besser zu verstehen und neue zu entdecken. Nur so kann man sein eigenes Forschungsprofil entwickeln.

\section{Der Druck verändert die Art der Forschung?}

Der Druck führt zu einer inkrementellen Arbeitsweise. Das ist bei uns nicht so ausgeprägt. Die jungen Leute in Deutschland riskieren mehr, weil sie nicht so unter Druck stehen. Sie können eher das Risiko eingehen, tiefe Resultate anzustreben. Wenn ich hier ein solches, tiefes Thema anbiete, sind die Promovenden immer sehr interessiert. Bei den chinesischen Doktoranden kommt schnell die Angst, Zeit zu verlieren. Dieser Unterschied ist nicht generisch, sondern gegeben durch die Jobsituation.

Wie funktioniert das, wenn diese Arbeitsweisen zusammenkommen, beispielsweise im Internationalen Graduierten Kolleg?

Das war anfangs durchaus schwierig. Zu einem solchen Internationalen Graduierten Kolleg gehören ja auch einige Austauschmonate. Durch den hohen Konkurrenzdruck hatten die chinesischen Studenten, die zu uns kamen, Angst, anderen von ihrer Arbeit zu erzählen.

Wie sind Sie damit umgegangen?

Wir haben hier sogenannte Cluster Groups. Das sind Arbeitsgruppen um ein Thema. Ich habe ihnen gesagt, das hat keinen Sinn hier, wenn ihr nicht offen erzählt, was ihr macht. Als ich merkte, warum sie davor Scheu hatten, habe ich scherzhaft hinzugefügt: Wenn auch nur ein einziger einem anderen das Thema wegschnappt - dem reiße ich den Kopf ab. Die haben mich völlig erschrocken angeschaut, als meinte ich das Ernst. Aber es hat funktioniert. 
Wir hatten eine gute, offene Arbeitsatmosphäre. Und es ist nichts passiert. Das lag aber auch an den Betreuern der Doktoranden in China, die das genauso gesehen haben. Es sind so wirklich exzellente Doktorarbeiten dabei herausgekommen.

Haben Sie umgekehrt Dinge in China kennengelernt, die Sie sich hier wünschen würden?

Die scharfe Konkurrenz hat auch eine zweite Seite. Die chinesischen Doktoranden arbeiten von morgens 6 bis abends 10, Wochenende gibt es nicht, und wenn man ein Buch für seine Arbeit braucht, dann kann man das in zwei Wochen durcharbeiten. Als unsere Doktoranden diesen Einsatz bei den Austauschdoktoranden gesehen haben, waren sie erst einmal erschrocken. Man muss sich natürlich fragen, wie effektiv eine so lange Arbeitszeit ist. Aber zu sehen, wie viel andere investieren, hat auch auf unsere Promovenden ein Stück abgefärbt. Das finde ich gut, denn mit einer Nine-To-Five-Mentalität kann man bei mir nicht promovieren.

Wie steht die deutsche Mathematik im internationalen Vergleich da?

Sicherlich sehr gut. Ich habe am Rande der gemeinsamen Tagung in Korea ein Interview gegeben, das die Situation vielleicht zusammenfasst: Die Journalistin befragte mich zum Mathematikunterricht in Deutschland. Ich erklärte ihr, dass in Deutschland an verschiedenen Stellen vereinfacht worden ist. Zum Beispiel, wenn nicht mehr gelehrt wird, wie man Beträge auflöst. Sie war völlig überrascht. Deutschland habe doch diese überragende Tradition und hohes Ansehen in der Mathematik. Deutschland ist in der höchsten IMU-Klasse. Warum sollten wir da nachlassen?

Wie beurteilen Sie die Situation des MathematikUnterrichts in Deutschland?

Ich hatte dazu vor Kurzem ein Telefonat mit Michael Meister. Er ist parlamentarischer Staatssekretär im BMBF und promovierter Mathematiker. Aus meiner persönlichen Erfahrung ist die Qualifikation der Studienanfänger schlechter geworden. Aber das sind nur persönliche Erfahrungen. Deshalb betone ich immer, dass wir dringend empirische Untersuchungen zum Mathematik-Unterricht benötigen. Bevor wir den Zeigefinger erheben, sollten wir mehr fundierte Information haben, um entscheiden zu können, ob das wirklich nur ein Problem des Mathematik-Unterrichts ist.

Sie erwähnen Michael Meister. Wie war die Zusammenarbeit mit der Politik?

Ich habe mich immer sehr gefreut, wenn Politiker auf mich als Präsident zugekommen sind. Und das war dann auch immer sehr fruchtbar, wie in dem erwähnten Fall. Andererseits dränge ich mich nicht gerne auf. Deshalb bin ich selten von mir aus auf Politiker zugegangen. Ich würde sagen, das war eine Schwäche meiner Präsidentschaft.
Was kann der Präsident der DMV überhaupt alleine erreichen? Letztlich ist es doch nur eine Person.

Das Wort des Präsidenten hat Gewicht. Es ist etwas völlig anderes, ob ich als Mathematik-Professor etwas sage oder als der Präsident der DMV. Und das muss man nutzen. Man darf es natürlich nicht übertreiben. Aber man braucht den Mut, als Präsident eine klare Aussage zu treffen - auch wenn man nicht mit letzter Gewissheit quod erat demonstrandum dahinter schreiben kann.

Wie beurteilen Sie das Verhältnis zwischen Mathematik und unserer Gesellschaft?

Seit dem Jahr der Mathematik ist dieses Verhältnis auf einem sehr guten Wege. Die Mathematik hat deutlich an Ansehen gewonnen. Früher traf man häufig auf Skepsis gegenüber dem, was die Mathematiker so machen. Heute weiß jeder, dass man die Mathematik unbedingt braucht. Dennoch glaube ich, dass wir alle Möglichkeiten nutzen sollten, dieses Verhältnis zu verbessern.

Wie kann man dazu beitragen, wenn man nicht gerade Präsident der DMV ist?

Man sollte die Chancen ergreifen, die sich einem zur Öffentlichkeitsarbeit bieten. Meine Erfahrung ist, dass mehr ankommt, als man erwartet. Wir hatten in Bielefeld eine große Tagung zur stochastischen Analysis. In dem $\mathrm{Zu}-$ sammenhang habe ich ein Interview für die Lokal Zeit ein regionales TV-Programm - gegeben. Ich habe in etwa Folgendes gesagt: Man kann mit stochastischen Methoden erklären, warum die gemäß dem Milankovič-Zykel alle 10 ooo Jahre auftretenden Eiszeiten etwa alle 100 ooo Jahre viel kälter sind. Im Nachhinein war ich hoch überrascht, dass fast alle meine Bekannten in der Region das Interview gesehen hatten und auf einmal Interesse an meiner Arbeit zeigten. Es kommt darauf an, dass man Emotion und Begeisterung für die eigene Arbeit zeigt.

Was waren für Sie die wichtigsten Themen in der Zeit Ihrer Präsidentschaft?

Vier Punkte stehen für mich im Vordergrund. Ein großes Thema ist der Übergang Schule-Hochschule. Ich bin froh, dass sich hier eine Dialektik entwickelt hat - insbesondere der Brandbrief und die Reaktionen - und dass wir von der Diskussion zu konstruktiven Vorschlägen kommen.

Sehr wichtig sind mir auch die Vorsemester. Hier haben wir uns erfolgreich bemüht, dass mehr Studierende die Kurse besuchen können.

Wirklich wertvoll ist auch die Zusammenarbeit unter den mathematisch-naturwissenschaftlichen Fachgesellschaften in Deutschland und die Treffen der europäischen und internationalen, mathematischen Gesellschaften. Beispielsweise in der Schuldiskussion hilft es, wenn man sich austauscht.

Die meiste Faszination ging für mich von der Nachwuchsarbeit aus. Dazu gehören der Bundeswettbewerb, die Olympiade aber auch noch viele andere Projekte. Das gibt 
es in diesem Ausmaß fast nur in Deutschland. Die Menschen, die das meist ehrenamtlich betreuen, bekommen viel zu wenig Kredit.

Wenn Sie sich zu Weihnachten etwas für die DMV wünschen könnten, was wäre das?

Ich würde mir eine breite Basis an aktiven Mitgliedern wünschen. Es gibt viele Mitglieder, die sich stark einbringen. Aber es sollten mehr sein. Denn häufig lässt sich, was Mitglieder zu Recht kritisieren, nicht aus dem Präsidium heraus beheben. Ein Beispiel sind die Jahrestagungen. Wir haben dazu eine Kommission eingerichtet. Aber letztlich muss die Kritik der Mitglieder selbst konstruktiv werden und zu einer aktiven Teilnahme an der DMV führen.

Ihre Präsidentschaft ist vorbei. Sie sind Sprecher des SFB „Taming uncertainty and profiting from randomness ..." - ich bekomme den ganzen Titel gar nicht in eine Frage.

Ja, die Titel der SFBs! Ich war sechs Jahre in der DFGSenatskommission für SFBs. Wann immer der Titel eines mathematischen Antrags vorgelesen wurde, konnte man die angespannten Gesichter der Kollegen aus den anderen Fächern sehen. Das war immer sehr technisch.

Und das wollten Sie ändern, indem Sie den Titel verlängern?

Wir wollten eine Kernaussage im Titel haben: Man muss die Unsicherheit in den Griff bekommen, um stärkere Modelle formulieren zu können, man kann aber auch vom Zufall profitieren. SPDGs sind zum Beispiel oft well posed, wenn es PDGs vielleicht nicht sind. Dann wurde der Titel erweitert, um die Bandbreite des Antrags besser abzubilden und um im Titel klar zu machen, in welches Fach der SFB gehört. Letzteres ist ein Wunsch der DFG.

Jetzt ist die Präsidentschaft vorbei. Wird es jetzt mal langweilig?

Sicher nicht! Zum einen bleiben mir noch eine Reihe Aufgaben für die DMV. Und zum anderen habe ich im Moment sieben und bald zehn Promovenden.

Sie betreiben stochastische Analysis. Woran forschen Sie aktuell?

Aktuell interessiert mich eine neue Forschungsrichtung zum Zusammenhang zwischen zwei meiner Forschungsschwerpunkte, stochastische (partielle) Differentialgleichungen (SPDG) und Fokker-Planck-Gleichungen (FPG).

Was ist eine Fokker-Planck-Gleichung?
Fokker-Planck-Gleichungen beschreiben die Entwicklung, also die zeitliche Veränderung, einer stochastischen Dichte.

Worin besteht der Zusammenhang mit stochastischen PDGs?

Der Zusammenhang zwischen beiden Gleichungen besteht darin, dass die Verteilungen des stochastischen Lösungsprozesses zu einem Zeitpunkt $t$ eine Lösung der zugehörigen Fokker-Planck-Gleichungen sind. Falls die Koeffizienten der SPDG nicht von den Zeitmarginalen des Lösungsprozesses abhängen, ist die zugehörige FPG linear und es ist ein fundamentales Resultat der stochastischen Analysis, dass dann unter recht allgemeinen Annahmen an die Koeffizienten SPDG und FPG mathematisch äquivalent sind.

Was heisst hier mathematisch äquivalent?

Äquivalent bedeutet hier, dass man nicht nur aus einer Lösung der SPDG eine Lösung der zugehörigen FPG erhält, sondern auch umgekehrt aus einer Lösung der FPG eine Lösung der SPDG konstruieren kann.

Und falls die Koeffizienten der SPDG doch von den Zeitmarginalen des Lösungsprozesses abhängen und die zugehörige FPG nicht mehr linear ist - was dann?

Das ist genau die Frage, die mich interessiert. Uns ist gerade die Konstruktion eines solchen stochastischen Prozesses gelungen, dessen Verteilung die Lösung der Poröse Medien-Gleichung ist. Unser allgemeines Ziel ist, die beschriebene volle Äquivalenz aus dem linearen Fall für eine SPDG mit marginalabhängigen Koeffizienten zu ihrer zugehörigen nichtlinearen FPG zu beweisen. Genauer gesagt suchen wir die Bedingungen, unter denen die Äquivalenz gilt.

Warum ist das so interessant?

Mit der Äquivalenz kann man stochastische Methoden zum Studium von nichtlinearen FPGs nutzen und umgekehrt analytische Methoden zum Studium von SPDEs verwenden. So ein Resultat gibt mir zum Einen eine Interpretation. Aber zum Anderen ermöglicht es zu approximieren. Das wiederum könnte, denke ich, numerisch sehr interessant werden. Aber bis dahin bleibt noch ein langer Weg.

Viel Erfolg und Freude dabei! 